\title{
Innovative solutions for storing infected plant samples.
}

\author{
Rania Sami Ammar and Damiana Haroun Khalil \\ Plant protection research Institute sabhya, Alexandria
}

Researchers face some problems during the collection and examination of samples, especially the leave ones, and the most important of which is to keep plant specimens infested with insects until they are investigated in the case of collecting numbers, as they reach a thousand to four thousand leaves, so we must examine this number on the same day to prevent leaving it because there are no distortions to it and to obtain to read closely the insects or the occurrence of damage to the paper and rot and control the movement of insects

In order to obtain an accurate reading, a solution was necessary to save the sample in some way to take the reading for a later time, especially taking a picture of the plant leaf and keeping it where there are many devices to take pictures with mobile or digital cameras and store them in a file on the computer in insects that are not seen by the naked eye . (Plat A)

As for the other type, there is difficulty in imaging due to the high speed of devices equipped with digital cameras, and the solution was to store such images with the least laboratory capabilities available in the laboratory, by installing the web camera and placing it on the eyepiece of a stereo microscope and fixing it on it with fixing it and connecting it to the computer that seen under the microscope became available on computer which stored with the date and number, and this process is done on the rest of the papers until they are saved at a later time. (Plat B) 
In order to facilitate the counting of insects, a program for the number of insects is being prepared automatically, thus reducing the time and effort for researchers to finish their laboratory work and facilitate such checks for non-workers in the agricultural field.

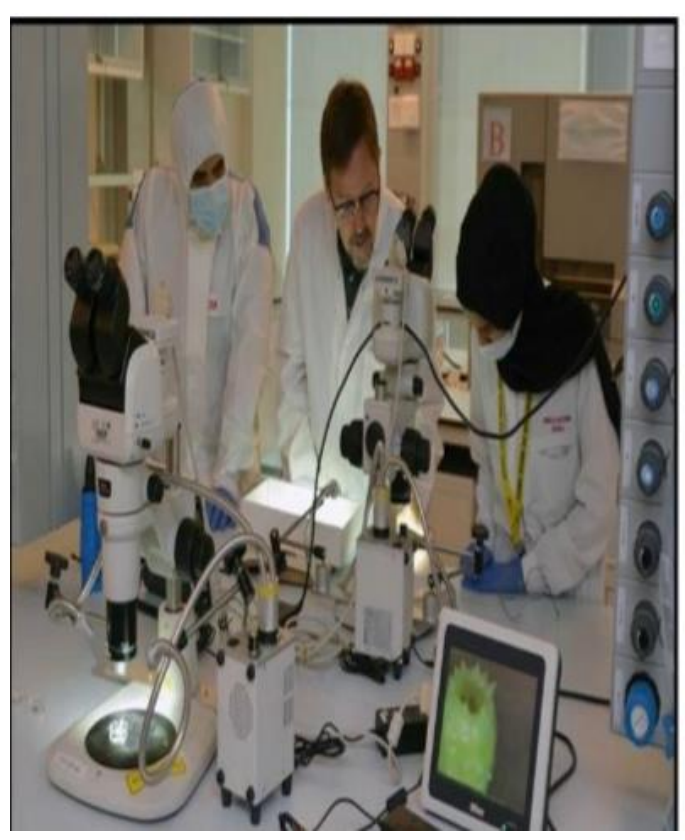

A

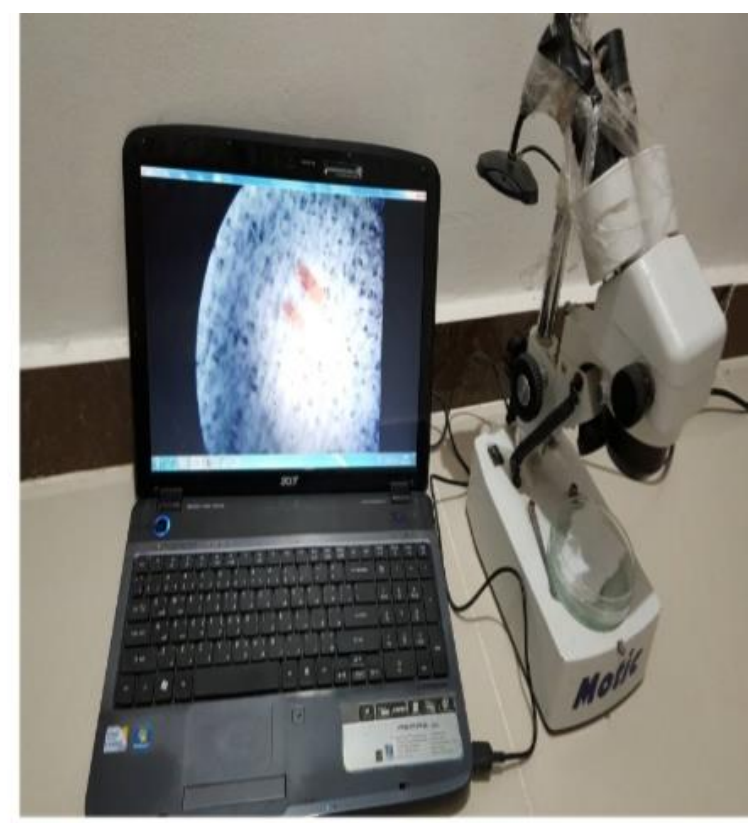

B 